\title{
Rough Neutrosophic Multisets Relation on Two Universal Sets
}

\author{
Suriana Alias ${ }^{1^{*}}$, Daud Mohamad ${ }^{2}$ and Adibah Shuib 3 \\ ${ }^{1}$ Department of Mathematics, Faculty of Computer and Mathematical Sciences, Universiti Teknologi \\ Mara (UiTM) Kelantan, Campus Machang, 18500 Machang, Kelantan, Malaysia \\ ${ }^{2,3}$ Department of Mathematics, Faculty of Computer and Mathematical Sciences, Universiti Teknologi \\ Mara (UiTM) Shah Alam, 40450 Shah Alam, Selangor, Malaysia
}

\begin{abstract}
Rough neutrosophic multisets relation over a universe has already been introduced as a notion of rough fuzzy relation theory over a universe. The concept of rough neutrosophic multisets relation is based on the definition of rough neutrosophic multisets. Essentially, rough neutrosophic multisets relation is a rough neutrosophic multisets in a Cartesian product of two universes. This paper introduces a rough neutrosophic multisets relation on two universal sets. The algebraic properties such as max, min, and composition of two rough neutrosophic multisets relation is examined. The inverse rough neutrosophic multisets relation is introduced and investigated. Finally, an application in medical diagnosis to investigate a patient's disease is presented by using this concept.
\end{abstract}

Keywords: Neutrosophic Set, Relation theory, Rough Neutrosophic Set, Rough Neutrosophic Multisets

\section{INTRODUCTION}

In the real-life situation, especially in decision analysis, there always involve the relation in the universal set to interpret the relations of the objects. For instance, in medical diagnosis, there exists a relation between symptoms set and diseases set, and in a staff selection problem there exists a relation between candidates set and attributes set. Samanta \& Sarkar (2011) have discussed the fuzzy rough relation over universe with their properties. Nguyen et al. (2014) extended that concept by introduced the rough fuzzy relations on the Cartesian product of two universe sets. Deli et al., (2015) have studied the relation on neutrosophic refined set with its properties. Arockiarani \& Antony (2016) introduced the rough neutrosophic relation on two universal sets. Nevertheless, at present, there is no study has been initiated on the rough neutrosophic multisets relation and mapping.
The elements of neutrosophic multisets (NM) introduced by Ye \& Ye (2014) is a sub element of neutrosophic refined set generalized by Broumi et al. (2014). The truth membership (T), indeterminate membership (I), and falsity membership (F) is refined to a sequence of $T_{1}, T_{2}, \ldots, T_{m}$ and $I_{1}, I_{2}, \ldots, I_{n}$ and $F_{1}, F_{2}, \ldots, F_{r}$ where $m, n, r \geq 1$. Then, Alias et al. (2017) introduced a rough neutrosophic multisets (RNM) as an approximation of imperfect knowledge represented by NM in a Pawlak's approximation space with equivalence relation.

Rough neutrosophic multisets relation (RNMR) gives further elaboration on the relationship between NM components which overcome the limitation of fuzzy multisets (FM) introduced by Miyamoto (2001) and intuitionistic fuzzy multisets (IFM) introduced by Shinoj \& John (2012). The FM cannot express the inconsistency of the information meanwhile the IFM is not able to handle the indeterminacy information, even though the multisets element is also allowed in FM and IFM theory.

In this paper, the generalization of rough fuzzy relation,

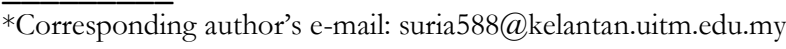


rough intuitionistic fuzzy relation and rough neutrosophic relation on two universals are defined, and their properties are examined. The remaining part of this paper is organized as follows. Next section elaborates some mathematical preliminary concepts of RNMR on two universal sets. The definition of RNMR on Cartesian product on two universal sets and related operations and properties are investigated. Finally, the application in medical diagnosis via RNMR theory is presented. The last section will conclude the finding of the paper.

\section{PRELIMINARIES}

In this section, the definition of the RNM, RNMR over a universe set were recall for more understanding about RNMR on two universal sets. All the proofs of properties and operations are shown in Alias et al. (2017); Alias et al. (2018).

Definition 1 (Rough Neutrosophic Multisets): Let $U$ be a non-null set and $R$ be an equivalence relation on $U$. Let $A$ be neutrosophic multisets in $U$ with the truthmembership sequence $T_{A}^{i}$, indeterminacy-membership sequences $I_{A}^{i}$ and falsity-membership sequences $F_{A}^{i}$. The lower and the upper approximations of $A$ in the approximation $(U, R)$ denoted by $\underline{N m}(A)$ and $\overline{N m}(A)$ are respectively defined as follows:

$N m(A)=$

$$
\left\{<x,\left(T_{\underline{N m}(A)}^{i}(x), I_{\underline{N m}(A)}^{i}(x), F_{\underline{N m}(A)}^{i}(x)\right)>\right.
$$

$\left.[x]_{R}, x \in U\right\}$

$\overline{N m}(A)=$

$$
\left\{<x,\left(T_{\overline{N m}(A)}^{i}(x), I_{\overline{N m}(A)}^{i}(x), F_{\overline{N m}(A)}^{i}(x)\right)>\quad \mid y \in\right.
$$

$\left.[x]_{R}, x \in U\right\}$

where

$i=1,2, \ldots, p$ and positive integer

$T_{\underline{N m}(A)}^{i}(x)=\Lambda_{y \in[x]_{R}} T_{A}^{i}(y), I_{\underline{N m}(A)}^{i}(x)=\vee_{y \in[x]_{R}} I_{A}^{i}(y)$,

$F_{\underline{N m}(A)}^{i}(x)=\bigvee_{y \in[x]_{R}} F_{A}^{i}(y)$,

$T_{\overline{N m}(A)}^{i}(x)=\bigvee_{y \in[x]_{R}} T_{A}^{i}(y), I_{N m(A)}^{i}(x)=\wedge_{y \in[x]_{R}} I_{A}^{i}(y)$,

$F_{\overline{N m}(A)}^{i}(x)=\bigwedge_{y \in[x]_{R}} F_{A}^{i}(y)$.

Here $\wedge$ and $\vee$ denote "min" and " $\max$ " operators respectively and $[x]_{R}$ is the equivalence class of the $x . T_{A}^{i}(y), I_{A}^{i}(y)$ and $F_{A}^{i}(y)$ are the membership sequences, indeterminacy sequences and non-membership sequences of $y$ with respect to $A$.

It is easy to see that $T_{\underline{N m}(A)}^{i}(x), I_{\underline{N m}(A)}^{i}(x), F_{\underline{N m}(A)}^{i}(x) \in$ $[0,1]$ and

$0 \leq T_{\underline{N m}(A)}^{i}(x)+I_{\underline{N m}(A)}^{i}(x)+F_{\underline{N m}(A)}^{i}(x) \leq 3$. Then, $\underline{N m}(A)$ is a neutrosophic multisets. Similarly, we have $T_{\overline{N m}_{(A)}^{i}}(x), I_{\overline{N m}(A)}^{i}(x), F_{\overline{N m}(A)}^{i}(x) \in[0,1]$ and $0 \leq T_{\overline{N m}(A)}^{i}(x)+I_{\overline{N m}(A)}^{i}(x)+F_{\overline{N m}(A)}^{i}(x) \leq 3$.

Then, $\overline{N m}(A)$ is neutrosophic multisets. Since $\underline{N m}(A)$ and $\overline{N m}(A)$ are two neutrosophic multisets in $U$, thus the neutrosophic multisets mappings $\underline{N m}, \overline{N m}: N m(U) \rightarrow$ $N m(U)$ are respectively referred as lower and upper rough neutrosophic multisets approximation operators and the pair of $(\underline{N m}(A), \overline{N m}(A))$ is called the rough neutrosophic multisets $(\mathrm{RNM})$ in $(U, R)$ respectively.

Definition 2 (Rough Neutrosophic Multisets Relation over Universe Set (RNMR)): Let $U$ be a non-empty set and $X$ and $Y$ be the RNM in $U$. We call $\Re \subseteq U \times U$ is a RNMR on $U \times U$ based on the $X \times Y$, where $X \times Y$ is characterized by truth-membership sequence $T_{\mathfrak{R}}^{i}$, indeterminacy-membership sequences $I_{\mathfrak{R}}^{i}$ and falsitymembership sequences $F_{\Re}^{i}$, defined as

$\Re=\left\{<(x, y),\left(T_{\mathfrak{R}}^{i}(x, y)\right)\left(I_{\mathfrak{R}}^{i}(x, y)\right),\left(F_{\mathfrak{R}}^{i}(x, y)\right)>:(x, y) \in\right.$

$U \times U\}$

with condition if it satisfies:

(1) i) $T_{\mathfrak{R}}^{i}(x, y)=1$ for all $(x, y) \in \underline{X \times Y}$ where $\underline{X \times Y}=$ $\underline{\mathcal{R}_{U}}(X) \times \underline{\mathcal{R}_{U}}(Y)$,

ii) $T_{\mathfrak{R}}^{i}(x, y)=0$, for all $(x, y) \in U \times U-\overline{X \times Y}$ where $\overline{X \times Y}=$ $\overline{\mathcal{R}}_{U}(X) \times \overline{\mathcal{R}}_{U}(Y)$,

iii) $0<T_{\mathfrak{R}}^{i}(x, y)<1$, for all $(x, y) \in \overline{X \times Y}-\underline{X \times Y}$.

(2) i) $I_{\mathfrak{R}}^{i}(x, y)=0$, for all $(x, y) \in \underline{X \times Y}$ where $\underline{X \times Y}=$ $\underline{\mathcal{R}_{U}}(X) \times \underline{\mathcal{R}_{U}}(Y)$,

ii) $I_{\mathfrak{R}}^{i}(x, y)=1$, for all $(x, y) \in U \times U-\overline{X \times Y}$ where $\overline{X \times Y}=$ $\overline{\mathcal{R}}_{U}(X) \times \overline{\mathcal{R}}_{U}(Y)$,

iii) $0<I_{\mathfrak{R}}^{i}(x, y)<1$, for all $(x, y) \in \overline{X \times Y}-\underline{X \times Y}$.

(3) i) $F_{\mathfrak{R}}^{i}(x, y)=0$, for all $(x, y) \in \underline{X \times Y}$ where $\underline{X \times Y}=$ $\underline{\mathcal{R}_{U}}(X) \times \underline{\mathcal{R}_{U}}(Y)$,

ii) $F_{\mathfrak{R}}^{i}(x, y)=1$, for all $(x, y) \in U \times U-\overline{X \times Y}$ where $\overline{X \times Y}=$ $\overline{\mathcal{R}}_{U}(X) \times \overline{\mathcal{R}}_{U}(Y)$,

iii) $0<F_{\Re}^{i}(x, y)<1$, for all $(x, y) \in \overline{X \times Y}-\underline{X \times Y}$. 


\section{ROUGH NEUTROSOPHIC MULTISETS RELATION ON TWO UNIVERSAL SETS}

In this section, the Cartesian product of two RNM on two universal sets is defined. Then, the RNMR on two universal sets is studied with their desired properties. In the following section, we only consider the case where T, I, $\mathrm{F}$ are refined into the same number of subcomponents 1 , $2, \ldots, p$, and $T_{A}^{i}, I_{A}^{i}$ and $F_{A}^{i}$ are single valued neutrosophic numbers.

Definition 3. The Cartesian product of two RNM $X$ and $Y$ is defined as

$$
\begin{aligned}
X \times Y & =\left\{<(x, y),\left(T_{X \times Y}^{i}(x, y)\right),\left(I_{X \times Y}^{i}(x, y)\right)\right. \\
,\left(F_{X \times Y}^{i}(x, y)\right) & >: x \in U, y \in V\}
\end{aligned}
$$

where

$$
\begin{aligned}
& T_{X \times Y}^{i}(x, y)=\min \left\{T_{X}^{i}(x), T_{Y}^{i}(y)\right\}, \\
& I_{X \times Y}^{i}(x, y)=\max \left\{I_{X}^{i}(x), I_{Y}^{i}(y)\right\}, \\
& F_{X \times Y}^{i}(x, y)=\max \left\{F_{X}^{i}(x), F_{Y}^{i}(y)\right\}, \\
& T_{X \times Y}^{i}, I_{X \times Y}^{i}, F_{X \times Y}^{i}: U \rightarrow[0,1], \quad \text { and } \quad i=1,2, \ldots, p .
\end{aligned}
$$

Suppose that $U, V$ are two non-empty universal sets and $\mathcal{R}_{U}, \mathcal{R}_{V}$ are equivalent relations on $U, V$ respectively. Then $\mathcal{R}=\mathcal{R}_{U} \times \mathcal{R}_{V}$ is an equivalence relation on $U \times V$ where $[(u, v)]_{\mathcal{R}}=\left\{(x, y) \in U \times V: x \in[u]_{\mathcal{R}_{U}}, y \in[v]_{\mathcal{R}_{V}}\right\}$

Definition 4. Let $X, Y$ be the RNM on $U, V$, respectively. We call $\Re \subseteq U \times V$ is a RNMR on $U \times V$ based on the $X \times$ $Y$, where $X \times Y$ is characterized by truth-membership sequence $T_{\Re}^{i}$, indeterminacy-membership sequences $I_{\mathfrak{R}}^{i}$ and falsity-membership sequences $F_{\Re}^{i}$, defined as

$\Re=\left\{\begin{array}{c}<(x, y),\left(T_{\mathfrak{R}}^{i}(x, y)\right),\left(I_{\mathfrak{R}}^{i}(x, y)\right) \\ ,\left(F_{\mathfrak{R}}^{i}(x, y)\right)>(x, y) \in U \times V\end{array}\right\}$

with condition if it satisfies:

(1) i) $T_{\mathfrak{R}}^{i}(x, y)=1$ for all $(x, y) \in \underline{X \times Y}$ where $\underline{X \times Y}=$ $\underline{\mathcal{R}_{U}}(X) \times \underline{\mathcal{R}_{V}}(Y)$,

ii) $T_{\mathfrak{R}}^{i}(x, y)=0$, for all $(x, y) \in U \times \mathrm{V}-\overline{X \times Y}$ where $\overline{X \times Y}=$ $\overline{\mathcal{R}}_{U}(X) \times \overline{\mathcal{R}}_{V}(Y)$

iii) $\quad 0<T_{\mathfrak{R}}^{i}(x, y)<1, \quad$ for $\quad$ all $\quad(x, y) \in \overline{X \times Y}-$ $\underline{X \times Y}$.

(2) i) $I_{\mathfrak{R}}^{i}(x, y)=0$, for all $(x, y) \in \underline{X \times Y}$ where $\underline{X \times Y}=$ $\underline{\mathcal{R}_{U}}(X) \times \underline{\mathcal{R}_{V}}(Y)$,

ii) $I_{\mathfrak{R}}^{i}(x, y)=1$, for all $(x, y) \in U \times \mathrm{V}-\overline{X \times Y}$ where $\overline{X \times Y}=$ $\overline{\mathcal{R}}_{U}(X) \times \overline{\mathcal{R}}_{V}(Y)$, iii) $0<I_{\mathfrak{R}}^{i}(x, y)<1$, for all $(x, y) \in \overline{X \times Y}-\quad \underline{X \times Y}$.

(3) i) $F_{\Re}^{i}(x, y)=0$, for all $(x, y) \in \underline{X \times Y}$ where $\underline{X \times Y}=$ $\underline{\mathcal{R}_{U}}(X) \times \underline{\mathcal{R}_{V}}(Y)$,

ii) $F_{\Re}^{i}(x, y)=1$, for all $(x, y) \in U \times \mathrm{V}-\overline{X \times Y} \quad$ where $\overline{X \times Y}=\overline{\mathcal{R}}_{U}(X) \times \overline{\mathcal{R}}_{V}(Y)$,

iii) $0<F_{\mathfrak{R}}^{i}(x, y)<1$, for all $(x, y) \in \overline{X \times Y}-$ $\underline{X \times Y}$.

\section{Remark 5 .}

(1) RNMR is also a relation on NM over a universe set. Therefore, RNMR follows the condition of relation on $\mathrm{NM}$, which is:

$T_{\mathfrak{R}}^{i}(x, y) \leq T_{X \times Y}^{i}(x, y), I_{\mathfrak{R}}^{i}(x, y) \geq I_{X \times Y}^{i}(x, y), F_{\mathfrak{R}}^{i}(x, y) \geq$ $F_{X \times Y}^{i}(x, y)$ for all $(x, y) \in U \times V$, and $0 \leq T_{\mathfrak{R}}^{i}(x, y)+$ $I_{\mathfrak{R}}^{i}(x, y)+F_{\Re}^{i}(x, y) \leq 3$.

(2) The generalization of relation for the rough neutrosophic set on two universal sets is obtained when $i=1$ for all element $T, I, F$ in definition 4, as follow;

$\Re=\left\{<(x, y),\left(T_{\mathfrak{R}}(x, y)\right)\left(I_{\mathfrak{R}}(x, y)\right),\left(F_{\mathfrak{R}}(x, y)\right)>:(x, y) \in\right.$ $U \times V\}$.

(3) The generalization of relation for rough intuitionistic fuzzy set on two universal sets obtained when $i=1$ for element $T$ and $F$, and properties (2) in definition 4 is omitted, as follow; $\Re=\left\{\left\langle(x, y),\left(T_{\mathfrak{R}}(x, y)\right),\left(F_{\mathfrak{R}}(x, y)\right)\right\rangle\right.$ $:(x, y) \in U \times V\}$.

(4) The generalization of the relation for rough fuzzy set of two universal sets is obtained when $i=1$ for element $T$ and properties (2) and (3) in definition 4 is omitted, as follow;

$\Re=\left\{<(x, y),\left(T_{\mathfrak{R}}(x, y)\right)>:(x, y) \in U \times V\right\}$.

The RNMR can be presented by relational tables and matrices likewise the representation of fuzzy relation (Nguyen et al. 2014). Since the triple $\left(T_{A}^{i}, I_{A}^{i}, F_{A}^{i}\right)$ has values in the interval $[0,1]$, the elements of the neutrosophic matrix also have values within $[0,1]$. Now, we consider some properties RNMR on two universal sets.

Proposition 6. Let $\Re_{1}, \Re_{2}$ be two $R N M R$ on $U \times V$ based on the $X \times Y$. Then $\Re_{1} \wedge \Re_{2}$ where

$$
\begin{aligned}
& T_{\mathfrak{R}_{1} \wedge \Re_{2}}^{i}(x, y)=\min \left\{T_{\Re_{1}}^{i}(x, y), T_{\mathfrak{R}_{2}}^{i}(x, y)\right\}, \\
& I_{\mathfrak{R}_{1} \wedge \Re_{2}}^{i}(x, y)=\max \left\{I_{\mathfrak{R}_{1}}^{i}(x, y), I_{\mathfrak{R}_{2}}^{i}(x, y)\right\}, \\
& \quad F_{\mathfrak{R}_{1} \wedge \Re_{2}}^{i}(x, y)=\max \left\{F_{\mathfrak{R}_{1}}^{i}(x, y), F_{\mathfrak{R}_{2}}^{i}(x, y)\right\}
\end{aligned}
$$

for all $(x, y) \in U \times V$, is a $R N M$ on $U \times V$ based on the 
$X \times Y$ and $i=1,2, \ldots, p$.

Proof. We show that $\Re_{1} \wedge \Re_{2}$ satisfies definition 4 .

Proposition 7. Let $\Re_{1}, \Re_{2}$ be two $R N M R$ on $U \times V$ based on the $X \times Y$. Then $\Re_{1} \vee \Re_{2}$ where

$$
\begin{aligned}
& T_{\mathfrak{R}_{1} \vee \Re_{2}}^{i}(x, y)=\max \left\{T_{\mathfrak{R}_{1}}^{i}(x, y), T_{\mathfrak{R}_{2}}^{i}(x, y)\right\}, \\
& I_{\mathfrak{R}_{1} \vee \Re_{2}}^{i}(x, y)=\min \left\{I_{\mathfrak{R}_{1}}^{i}(x, y), I_{\mathfrak{R}_{2}}^{i}(x, y)\right\}, \quad F_{\mathfrak{R}_{1} \vee \Re_{2}}^{i}(x, y)= \\
& \min \left\{F_{\mathfrak{R}_{1}}^{i}(x, y), F_{\mathfrak{R}_{2}}^{i}(x, y)\right.
\end{aligned}
$$

for all $(x, y) \in U \times V$, is a $R N M$ on $U \times V$ based on the $X \times Y$ and $i=1,2, \ldots, p$.

The following properties of RNMR are obtained by using algebraic results.

Proposition 8. Let $\Re_{1}, \Re_{2}$ be two $R N M R$ on $U \times V$ based on the $X \times Y$. Then $\mathfrak{R}_{1} \otimes \Re_{2}$ where

$$
\begin{aligned}
& T_{\mathfrak{R}_{1} \otimes \Re_{2}}^{i}(x, y)=T_{\mathfrak{R}_{1}}^{i}(x, y) \cdot T_{\mathfrak{R}_{2}}^{i}(x, y), \\
& I_{\mathfrak{R}_{1} \otimes \Re_{2}}^{i}(x, y)=I_{\mathfrak{R}_{1}}^{i}(x, y)+I_{\mathfrak{R}_{2}}^{i}(x, y)-I_{\mathfrak{R}_{1}}^{i}(x, y) \cdot I_{\mathfrak{R}_{2}}^{i}(x, y), \\
& F_{\mathfrak{R}_{1} \otimes \Re_{2}}^{i}(x, y)=F_{\mathfrak{R}_{1}}^{i}(x, y)+F_{\mathfrak{R}_{2}}^{i}(x, y)-F_{\mathfrak{R}_{1}}^{i}(x, y) \cdot F_{\mathfrak{R}_{2}}^{i}(x, y) .
\end{aligned}
$$

for all $(x, y) \in U \times V$, is a $R N M$ on $U \times V$ based on the $X \times Y$ and $i=1,2, \ldots, p$.

Proof. The relation $\mathfrak{R}_{1} \otimes \mathfrak{R}_{2}$ satisfies definition 4 .

Proposition 9. Let $\Re_{1}, \Re_{2}$ be two $R N M R$ on $U \times V$ based on the $X \times Y$. Then $\mathfrak{R}_{1} \oplus \Re_{2}$ where

$$
\begin{gathered}
T_{\mathfrak{R}_{1} \oplus \Re_{2}}^{i}(x, y)=T_{\mathfrak{R}_{1}}^{i}(x, y)+T_{\mathfrak{R}_{2}}^{i}(x, y)-T_{\mathfrak{R}_{1}}^{i}(x, y) \cdot T_{\mathfrak{R}_{2}}^{i}(x, y), \\
I_{\mathfrak{R}_{1} \oplus \Re_{2}}^{i}(x, y)=I_{\mathfrak{R}_{1}}^{i}(x, y) \cdot I_{\mathfrak{R}_{2}}^{i}(x, y), \\
\quad F_{\Re_{1} \oplus \Re_{2}}^{i}(x, y)=F_{\Re_{1}}^{i}(x, y) \cdot F_{\mathfrak{R}_{2}}^{i}(x, y)
\end{gathered}
$$

for all $(x, y) \in U \times V$, is a $R N M$ on $U \times V$ based on the $X \times Y$ and $i=1,2, \ldots, p$.

Proof. The relation $\mathfrak{R}_{1} \oplus \mathfrak{R}_{2}$ satisfies definition 4 .

\section{A. Composition of Two Rough Neutrosophic Multisets Relation}

Let $U, V, W$ be the universal sets and $\Re_{1}, \Re_{2}$ are two RNMR on $U \times V, V \times W$ based on $X \times Y, Y \times Z$, respectively.

Definition 1o. Composition of two RNMR $\mathfrak{R}_{1}, \mathfrak{R}_{2}$ denoted $\Re_{1} \circ \Re_{2}$ which defined on $U \times W$ based on $X \times Z$ where $T_{\mathfrak{R}_{1} \circ \Re_{2}}^{i}(x, z)=\max _{y \in V}\left\{\min \left[T_{\mathfrak{R}_{1}}^{i}(x, y), T_{\mathfrak{R}_{2}}^{i}(y, z)\right]\right\}$, $I_{\mathfrak{R}_{1} \circ \Re_{2}}^{i}(x, z)=\min _{y \in V}\left\{\max \left[I_{\mathfrak{R}_{1}}^{i}(x, y), I_{\mathfrak{R}_{2}}^{i}(y, z)\right]\right\}$, $F_{\Re_{1} \circ \Re_{2}}^{i}(x, z)=\min _{y \in V}\left\{\max \left[F_{\Re_{1}}^{i}(x, y), F_{\Re_{2}}^{i}(y, z)\right]\right\}$ for all $(x, z) \in U \times W$ and $i=1,2, \ldots, p$.

Proposition 11. $\Re_{1} \circ \Re_{2}$ is a $R N M R$ on $U \times W$ based on $X \times Z$.
Proof.

1 i) Since $\Re_{1}, \Re_{2}$ are two RNMR on $U \times V, V \times W$ based on $X \times Y, Y \times Z$ respectively, then $T_{\mathfrak{R}_{1}}^{i}(x, y), T_{\mathfrak{R}_{2}}^{i}(y, z)=1$ for all $(x, y) \in \underline{X \times Y}$ and $(y, z) \in \underline{Y \times Z}$. We denote $\underline{Y}=\mathcal{R}_{V}(Y)$ and we have

$$
\begin{gathered}
T_{\mathfrak{R}_{1} \circ \Re_{2}}^{i}(x, z)=\max _{y \in V}\left\{\min _{(x, z) \in \underline{X \times Z}}\left[T_{\mathfrak{R}_{1}}^{i}(x, y), T_{\mathfrak{R}_{2}}^{i}(y, z)\right]\right\} \\
=\max _{y \in \underline{Y}}\left\{\min _{(x, z) \in \underline{X \times Z}}\left[T_{\mathfrak{R}_{1}}^{i}(x, y), T_{\mathfrak{R}_{2}}^{i}(y, z)\right]\right\} \\
\mathrm{V} \max _{v \in V-\underline{Y}}\left\{\min _{(x, z) \in \underline{X \times Z}}\left[T_{\mathfrak{R}_{1}}^{i}(x, v), T_{\mathfrak{R}_{2}}^{i}(v, z)\right]\right\}=1 \\
=\mathrm{V} \max _{v \in V-\underline{Y}}\left\{\min _{(x, z) \in \underline{X \times Z}}\left[T_{\mathfrak{R}_{1}}^{i}(x, v), T_{\mathfrak{R}_{2}}^{i}(v, z)\right]\right\} \\
=1 \text { for all }(x, z) \in \underline{X \times Z} . \\
\text { ii) Note that } T_{\mathfrak{R}_{1}}^{i}(x, y), T_{\mathfrak{R}_{2}}^{i}(y, z)=0 \text { for all }(x, y) \in U \times V-
\end{gathered}
$$
$\overline{X \times Y} \quad$ and $\quad(y, z) \in V \times W-\overline{Y \times Z}$. We consider $T_{\mathfrak{R}_{1} \circ \Re_{2}}^{i}(x, z)=$ $\max _{y \in V}\left\{\min _{(x, z) \in U \times W-\overline{X \times Z}}\left[T_{\mathfrak{R}_{1}}^{i}(x, y), T_{\mathfrak{R}_{2}}^{i}(y, z)\right]\right\}$.

For all $(x, z) \in U \times W-\overline{X \times Z}$, it exists $x \notin \overline{\mathcal{R}_{U}}(X)$ so $(x, v) \in$ $U \times V-\overline{X \times Y}$ and $T_{\Re_{1}}^{i}(x, v)=0$ for all $v \in V$. Similarly, there exists $z \notin \overline{\mathcal{R}_{U}}(Z)$ such that $(v, z) \in V \times W-\overline{Y \times Z}$ and $T_{\mathfrak{R}_{2}}^{i}(v, z)=0$ for all $v \in V$.

Hence $\min _{(x, z) \in U \times W-\overline{X \times Z}}\left[T_{\mathfrak{R}_{1}}^{i}(x, v), T_{\mathfrak{R}_{2}}^{i}(v, z)\right]=0$ for all $(x, z) \in U \times W-\overline{X \times Z}$ and $v \in$ and thus,

$T_{\Re_{1} \circ \Re_{2}}^{i}(x, z)=$ $\max _{y \in V}\left\{\min _{(x, z) \in U \times W-\overline{X \times Z}}\left[T_{\mathfrak{R}_{1}}^{i}(x, y), T_{\mathfrak{R}_{2}}^{i}(y, z)\right]\right\}=0$ for all $(x, z) \in U \times W-\overline{X \times Z}$.

iii) We must proof

$0<\max _{y \in V}\left\{\min \left[T_{\mathfrak{R}_{1}}^{i}(x, y), T_{\mathfrak{R}_{2}}^{i}(y, z)\right]\right\}<1$, for all $(x, z) \in$ $\overline{X \times Z}-\underline{X \times Z}$. Since $(x, z) \notin \underline{X \times Z}$, then there exists $x \notin$ $\underline{X}=\underline{\mathcal{R}_{U}}(X) \quad$ or $\quad Z \notin \underline{Z}=\underline{\mathcal{R}_{W}}(Z) \quad$ such that $\min \left[T_{\mathfrak{R}_{1}}^{i}(x, y), T_{\mathfrak{R}_{2}}^{i}(y, z)\right]<1$ for all $y \in Y$. On the other hand, $T_{\mathfrak{R}_{1}}^{i}(x, v)=T_{\mathfrak{R}_{2}}^{i}(v, z)=0$ for all $(x, z) \in U \times W-\overline{X \times Z}$ and $v \in V$ then, we have $0<\left\{\min \left[T_{\mathfrak{R}_{1}}^{i}(x, y), T_{\mathfrak{R}_{2}}^{i}(y, z)\right]\right\}<1$ for all $(x, z) \in \overline{X \times Z}-\underline{X \times Z}$ and $y \in Y$. Hence, we have $0<\max _{y \in V}\left\{\min \left[T_{\mathfrak{R}_{1}}^{i}(x, y), T_{\mathfrak{R}_{2}}^{i}(y, z)\right]\right\}<1$ or $0<T_{\mathfrak{R}_{1} \circ \Re_{2}}^{i}(x, z)<1$ for all $(x, z) \in \overline{X \times Z}-\underline{X \times Z}$. The proof of 2) and 3 ) are is similar to 1).

Note that $\mathfrak{R}_{1} \circ \mathfrak{R}_{2} \neq \mathfrak{R}_{2} \circ \mathfrak{R}_{1}$, since the composition of two RNMR $\Re_{1}, \Re_{2}$ exists but the composition of two RNMR $\mathfrak{R}_{2}, \mathfrak{R}_{1}$ does not necessarily exist. 


\section{B. Inverse Rough Neutrosophic Multisets Relation}

Let $X$ and $Y$ be the two RNM on $U$ and $V$, respectively. $\Re \subseteq U \times V$ is a RNMR on $U \times V$ based on the $X \times Y$. Then we define $\mathfrak{R}^{-1} \subseteq V \times U$ is the RNMR on $V \times U$ based on $Y \times X$ as following:

$\mathfrak{R}^{-1}=\left\{\begin{array}{c}<(y, x),\left(T_{\mathfrak{R}^{-1}}^{i}(y, x)\right),\left(I_{\mathfrak{R}^{-1}}^{i}(y, x)\right), \\ \left(F_{\mathfrak{R}^{-1}}^{i}(y, x)\right)>:(y, x) \in V \times U\end{array}\right\}$

where $\quad T_{\mathfrak{R}^{-1}}^{i}(y, x)=T_{\mathfrak{R}^{i}}^{i}(x, y), \quad I_{\mathfrak{R}^{-1}}^{i}(y, x)=I_{\mathfrak{R}}^{i}(x, y)$, $F_{\mathfrak{R}^{-1}}^{i}(y, x)=F_{\Re}^{i}(x, y)$ for all $(y, x) \in V \times U$ and

$i=1,2, \ldots, p$.

Definition 12. The relation $\mathfrak{R}^{-1}$ is called the inverse RNMR of $\Re$.

Proposition 13. $\left(\mathfrak{R}^{-1}\right)^{-1}=\mathfrak{R}$.

Proof. The relation $\mathfrak{R}^{-1}$ satisfies definition 4.

Proposition 14. Let $\Re_{1}, \Re_{2}$ be two RNMR on $U \times V, V \times$ $W$ based on $X \times Y, Y \times Z$, respectively. Then $\left(\Re_{1}\right.$ 。 $\left.\mathfrak{R}_{2}\right)^{-1}=\mathfrak{R}_{2}^{-1} \circ \mathfrak{R}_{1}{ }^{-1}$.

Proof. For all $x \in U, y \in V, z \in W$, we have

$T_{\left(\Re_{1} \circ \Re_{2}\right)^{-1}}^{i}(z, x)=T_{\Re_{1} \circ \Re_{2}}^{i}(x, z)$

$=\max _{y \in V}\left\{\min \left[T_{\mathfrak{R}_{1}}^{i}(x, y), T_{\mathfrak{R}_{2}}^{i}(y, z)\right]\right\}$

$=\max _{y \in V}\left\{\min \left[T_{\left(\Re_{1}\right)^{-1}}^{i}(y, x), T_{\left(\Re_{2}\right)^{-1}}^{i}(z, y)\right]\right\}$

$=\max _{y \in V}\left\{\min \left[T_{\left(\Re_{2}\right)^{-1}}^{i}(z, y), T_{\left(\Re_{1}\right)^{-1}}^{i}(y, x)\right]\right\}=$

$T_{\left(\Re_{2}\right)^{-1} \circ\left(\Re_{1}\right)^{-1}(z, x) ;}^{i}$

The proofs for $I_{A}^{i}$ and $F_{A}^{i}$ are similar to $T_{A}^{i}$.

The inverse RNMR $\mathfrak{R}^{-1}$ can be represented by using matrix $M(\Re)^{t}$, the transposition of matrix $M(\Re)$.

\section{Medical Diagnosis via Rough Neutrosophic Multisets Relation Theory}

In medical diagnosis, symptoms will give a hint for patient suffering from a disease. For example, patients who suffer from Influenza will have the symptoms like a runny nose, sniffling, sore throat, fever, throbbing muscles, chills and sweats, and migraine (Anon 2018).

In this section, the relationship between the diseases and symptoms is modelled via rough neutrosophic multisets relation on two universal sets. Table 1 shows the example of medical findings of a patient represented in a tabular form. The three-time interval was recorded to obtain the best diagnosis of the patient's disease.

Table 1. Example of medical findings of Patient A

\begin{tabular}{|c|c|c|c|c|c|}
\hline $\begin{array}{l}\text { Tim } \\
\text {-ing }\end{array}$ & $\begin{array}{l}\text { Temp } \\
\text { e- } \\
\text { rature }\end{array}$ & $\begin{array}{l}\text { Hea } \\
\text { d- } \\
\text { ach } \\
\text { e }\end{array}$ & $\begin{array}{l}\text { Vo- } \\
\text { miti } \\
\text { ng }\end{array}$ & $\begin{array}{l}\text { Swe } \\
- \\
\text { atin } \\
\text { g }\end{array}$ & $\begin{array}{l}\text { Che } \\
\text { ss } \\
\text { pai } \\
\text { n }\end{array}$ \\
\hline $\begin{array}{l}6: 0 \\
0 \\
a \mathrm{am}\end{array}$ & $\begin{array}{l}100.8 \\
\text { oF }\end{array}$ & $\begin{array}{l}\text { Yes } \\
\text { (mo } \\
\text { der } \\
\text { ate) }\end{array}$ & No & $\begin{array}{l}\text { Yes } \\
\text { (hig } \\
\text { h) }\end{array}$ & $\begin{array}{l}\text { Yes } \\
\text { (hig } \\
\text { h) }\end{array}$ \\
\hline $\begin{array}{l}\text { 12:0 } \\
\text { o } \\
\text { pm }\end{array}$ & $\begin{array}{l}97.4^{\circ} \\
\mathrm{F}\end{array}$ & $\begin{array}{l}\text { Yes } \\
\text { (hig } \\
\text { h) }\end{array}$ & $\begin{array}{l}\text { Yes } \\
\text { (mo } \\
\text { der } \\
\text { ate) }\end{array}$ & No & $\begin{array}{l}\text { Yes } \\
\text { (mo } \\
\text { der } \\
\text { ate) }\end{array}$ \\
\hline $\begin{array}{l}6: 0 \\
0 \\
\text { pm }\end{array}$ & $\begin{array}{l}100.4 \\
\text { oF }\end{array}$ & $\begin{array}{l}\text { Yes } \\
\text { (mo } \\
\text { der } \\
\text { ate) }\end{array}$ & $\begin{array}{l}\text { Yes } \\
\text { (mo } \\
\text { der } \\
\text { ate) }\end{array}$ & $\begin{array}{l}\text { Yes } \\
\text { (mo } \\
\text { der } \\
\text { ate) }\end{array}$ & $\begin{array}{l}\text { Yes } \\
\text { (mo } \\
\text { der } \\
\text { ate) }\end{array}$ \\
\hline
\end{tabular}

All the medical findings are transformed into RNM representations.

Let $U=\left\{d_{1}, d_{2}, d_{3}\right\}$ be a set of three diseases, where $d_{i}(i=1,2,3)$ represent "malaria, typhoid fever, and influenza", and the universe $V=\left\{s_{1}, s_{2}, s_{3}, s_{4}, s_{5}\right\}$ be a set of five symptoms, where $s_{j}(j=1,2,3,4,5)$ represents "temperature, headache, vomiting, sweating, and chess pain”.

According to experts, typhoid fever and influenza can be stated in the same contingent of disease and headache, while chest pain and vomiting are in the same contingent of symptoms. The relationship between the disease $d_{i}\left(d_{i} \in U\right)$ and the symptom $s_{j}\left(s_{j} \in V\right)$ is represented by RNMR $\Re$ from $U$ to $V$. From the medical data for patient A, RNM, $D$ and $S$ on $U, V$ respectively, are obtained at different time intervals such as 6:00 am, 12:00 and 6:00 pm as follows:

$$
\begin{aligned}
D=\{ & <d_{1},(1.0,0.3,0.5),(0.4,0.5,1.0),(0.6,0.8,0.8)>, \\
& <d_{2},(0.5,0.7,1.0),(0.3,0.5,1.0),(0.6,0.7,0.8)>, \\
& \left.<d_{3},(1.0,0.6,0.4),(0.5,0.6,0.7),(0.5,0.6,0.8)>\right\}
\end{aligned}
$$

and

$$
\begin{aligned}
S=\{ & <s_{1},(0.6,0.8,1.0),(0.5,0.7,1.0),(0.3,0.9,0.6)>, \\
& <s_{2},(0.7,0.8,0.9),(0.3,1.0,0.8),(0.5,0.6,0.7)>, \\
& <s_{3},(1.0,0.9,1.0),(0.4,0.7,0.9),(0.5,0.9,1.0)>, \\
& <s_{4},(1.0,0.9,0.9),(0.4,0.9,0.9),(0.6,0.7,0.8)>, \\
& \left.<s_{5},(1.0,0.7,1.0),(0.3,0.6,0.8),(0.7,1.0,0.8)>\right\} .
\end{aligned}
$$

By satisfying all the conditions in definition 4 , the disease of Patient A is diagnosed by defining the relation of RNM $\Re$ on diseases and symptoms $U \times V$ based on $D \times S$. All steps are followed as a relation of RNM $\Re$ over a 
universe (Alias et al., 2018).

Table 2. Score of three diseases for Patient A

\begin{tabular}{cc|cc}
\hline & Score & & Score \\
\hline $\mathfrak{R}_{\mathbf{1}}$ & & $\mathfrak{R}_{2}$ & \\
$d_{1}$ & $\boldsymbol{o . 9}$ & $d_{1}$ & 0.3 \\
$d_{2}$ & $o .8$ & $d_{2}$ & 0.5 \\
$d_{3}$ & 0.7 & $d_{3}$ & $\boldsymbol{o . 7}$ \\
\hline $\mathfrak{R}_{3}$ & & $\mathfrak{R}_{4}$ & \\
$d_{1}$ & $\boldsymbol{o . 8}$ & $d_{1}$ & 0.6 \\
$d_{2}$ & $\boldsymbol{o . 8}$ & $d_{2}$ & 0.7 \\
$d_{3}$ & $\boldsymbol{o . 8}$ & $d_{3}$ & $\boldsymbol{o . 8}$ \\
\hline
\end{tabular}

From the result scores of four possible relations of disease and symptoms, it shows that very likely Patient A is suffering from Influenza.

When compared with the approaches of weighted similarity measures by Chatterjee et al. (2015) and dice similarity measure by Ye \& Ye (2014) based on NM component, the result concludes that the patient was suffering from Influenza and Typhoid, respectively. The proposed approach based on RNMR gives a significant value for the relationship between the NM information, since the relation of RNM considers uncertain information by introducing the expert's opinion about the relation between each element in NM and interpreting them into lower and upper approximation.

\section{CONCLUSION}

A RNMR on two universal sets was first defined by their properties and operations such as max, min, the composition of two RNM, and inverse rough neutrosophic multisets. The application of RNMR theory to medical diagnosis had been successfully presented in this paper. Future works arising from this study will focus on other RNM properties, especially that lead to the development of decision-making process and methods.

\section{ACKNOWLEDGEMENT}

The authors would like to acknowledge the Faculty of Computer and Mathematical Sciences, Universiti Teknologi MARA (UiTM) and the Ministry of Higher Education, Malaysia, for their financial support. 


\section{REFERENCES}

Alias, S., Mohamad, D. \& Shuib, A. (2017). Rough Neutrosophic Multisets. Neutrosophic Sets and Systems, 16, 80-88.

Alias, S., Mohamad, D. \& Shuib, A. (2018). Rough Neutrosophic Multisets Relation with Application in Marketing Strategy. Neutrosophic Sets and Systems, 21, 36-55.

Anon, (2018). Symptom and Disease. viewed 6 September $2018<$ https://www.mayoclinic.org/diseasesconditions/flu/symptoms-causes.html >

Arockiarani, I. \& Antony Crispin Sweety, C. (2016). Rough Neutrosophic Relation on Two Universal Sets. Bulletin of Mathematics and Statistics Research, 3(4), 77-89.

Broumi, S., Deli, I. \& Smarandache, F. (2014). Neutrosophic Refined Relations and Their Properties. Neutrosophic Theory and Its Applications, 228-248.

Chatterjee, R., Majumdar, P. \& Samanta, S.K. (2015). Single valued neutrosophic multisets. Annals of Fuzzy Mathematics and Informatics, $\mathrm{x}(\mathrm{x}), 499-514$.

Miyamoto, S. (2001). Fuzzy multisets and their generalizations. Multiset Processing: Mathematical, Computer Science, and Molecular Computing Points of View, 2235, 225-235.

Nguyen, X.T., Nguyen, V.D. \& Nguyen, D.D. (2014). Rough Fuzzy Relation on Two Universal Sets. International Journal of Intelligent Systems and Applications, 6(4), 49-55.

Samanta, T.K. \& Sarkar, B. (2011). Fuzzy Rough Relations. arxiv:1109.1803, 1, 10-17.

Shinoj, T.K. \& John, S.J., (2012). Intuitionistic Fuzzy Multisets And Its Application in Medical Diagnosis. World Academy of Science, Engineering and Technology, 6(1), 1418-1421.

Ye, S. \& Ye, J. (2014). Dice similarity measure between single valued neutrosophic multisets and its application in medical diagnosis. Neutrosophic Sets and Systems, 6, 48-53. 